\title{
Capítulo 8 \\ Reflexiones metodológicas para investigar la comunicación no verbal
}

\author{
Cyntia Cerón Hernández* \\ UNIVERSIDAD INTERCONTINENTAL
}

Uno de los principales aspectos relevantes en el momento de desarrollar un proyecto de investigación en torno a la Comunicación no verbal, es la forma de acercarse a este objeto de estudio y generar conocimiento en torno a él. Este texto surge como reflexión acerca del diseño de la metodología idónea ante la complejidad que implica estudiar la Comunicación no verbal y abre así una posibilidad a nivel metodológico para estudiar un objeto de entrada poco abordado. Este trabajo está dividido en dos partes: por un lado hace una revisión teórica acerca de la metodología utilizada en este tipo de investigaciones desde diferentes disciplinas, y en la segunda parte se realiza una propuesta metodológica desde la Comunicación para realizar proyectos de investigación sobre la dimensión no verbal, integrando el nivel discursivo de los sujetos de estudio y la intervención en procesos comunicativos.

One of the main aspects at the moment for developing a research project around the nonverbal Communication is the form to approach this study object and the way to generate knowledge around it. This text arises as a reflection about the design of the suitable methodology regarding the complexity that implies to study the nonverbal Communication, and therefore

* Licenciada en Ciencias de la Comunicación por la Universidad Intercontinental con Especialidad en Políticas Culturales y Gestión Cultural por la Universidad Autónoma de México, el Centro Nacional de las Artes y la Organización de Estados Iberoamericanos. Ha trabajado en investigación de mercado a nivel cualitativo y actualmente es investigadora y profesora de la Escuela de Comunicación de la Universidad Intercontinental, Unidad Tlalpan, Ciudad de México. 
opens one methodological possibility to study a little boarded subject. This work is divided in two parts: on the one hand, it makes a theoretical revision about the methodology used in this type of researches from different disciplines, and in the second part a methodological proposal is made from the Communication to make research projects on the nonverbal dimension of the Communication, integrating the individuals' discourse and the intervention on communicative processes.

\section{INTRODUCCIÓN}

El término de Comunicación no verbal ha heredado del término comunicación la susceptibilidad de ser interpretado como ciencia o como objeto de estudio desde diferentes disciplinas y enfoques, razón por la cual muchas veces resulta complejo definir claramente sus alcances y límites. En ambos casos y en comparación con otras ciencias, los estudios relacionados con la Comunicación no verbal tienen relativamente poco tiempo y el uso de metodologías, instrumentos de análisis o resultados han generado controversia y han sido motivo de discusión; principalmente desde disciplinas con fuerte trayectoria y sustento teórico como la psicología o antropología ante algunas interpretaciones realizadas de forma universal y simplista que intentaron vender por ejemplo, recetas de éxito en las relaciones interpersonales.

Alrededor de este término se ha generado confusión ya que, desde la mirada dicotómica de la cultura occidental, se ha generado el mito acerca de la concepción de la Comunicación no verbal como una disección independiente de la comunicación humana, (al cual hace referencia Mark L. Knapp, 1982), cuando están íntimamente ligadas. Por otro lado, como menciona el mismo autor, al término no verbal se le asocia con fenómenos -no acústicos y no vocales- como una categoría diferente de -lo verbal, acústico y vocal-, cuando esta distinción no es necesariamente válida en todos los casos, ya que:

a) No todos los fenómenos acústicos son vocales como por ejemplo el aplaudir,

b) Existen fenómenos no acústicos que pueden ser verbales como el lenguaje de sordos,

c) Existen fenómenos vocales que no se vinculan necesariamente con lo verbal, sino que son respiratorios como suspirar o inspirar,

d) Existen palabras que no son claramente verbales por ejemplo el cuchichear o murmurar, 
e) Existen fenómenos vocales que no son verbales como sonidos con la lengua o los labios (aportación propia).

Asimismo, al considerar la Comunicación no verbal como tema de investigación, no queda claro si el objeto de estudio es la señal producida o si se hace referencia al código de interpretación de la señal. Por lo anterior, considero relevante conceptualizar la Comunicación no verbal como un sistema de códigos compartido dentro del fenómeno global y complejo de la comunicación humana que contempla el proceso de construcción, expresión y significación de todo aquello que tiene que ver con el lenguaje corporal en momentos de interacción en un lugar y tiempo determinados.

A continuación se hará una revisión alrededor de la Comunicación no verbal, como objeto de estudio y en relación a las metodologías que han sido utilizadas, para después llegar a una propuesta metodológica que aborda el tema en cuestión.

\section{ANTECEDENTES DE LA INVESTIGACión SOBRe COMUNiCACIÓN NO VERBAL}

La Comunicación no verbal ha sido objeto de estudio de diferentes disciplinas e intenciones desde la antropología, la semiótica, la oratoria o incluso la danza; razón por la cual la concepción del término y la visión en torno al tema se ha ido adaptando según el marco epistemológico que lo aborde. A pesar de este escenario, la investigación de la Comunicación no verbal es fruto principalmente de seis disciplinas: la Psicología, la Psiquiatría, la Antropología, la Sociología, la Semiótica y la Etología.

Los etólogos comenzaron a estudiar la expresión corporal al descubrir las similitudes entre el comportamiento humano y el comportamiento animal en situaciones de cortejo, dominio, crianza, sumisión, conciliación, etcétera. El primer estudio al que se hace referencia es "The expression of emotion in man and animals", escrito por Darwin en el año de 1872. Este trabajo aborda las expresiones faciales de mamíferos, incluido el hombre, y sugiere que todas las expresiones humanas primarias pueden remontarse a algún acto funcional primitivo.

La Psicología y la Psiquiatría son algunas de las fuentes de estudio que han tenido una fuerte contribución al conocimiento de la Comunicación no verbal. El interés por investigar este tema surgió de la necesidad por comprender la conducta humana al reconocer que la expresión corporal arroja indicaciones sobre conductas, emociones, personalidad de los sujetos, así como formas de interacción con los otros. En sus principios, psicólogos como Kretschmer 
(1925) con su publicación "Physique and character" y Sheldon (1940) con "The Variations of Human Physique" sentaron bases de tipos corporales y se interesaron en cómo se comunica la gente por las expresiones del rostro. En el año de 1941 David Efron escribe "Gesture and Environment", donde establece que la cultura es un aspecto importante en la formación de gestos y así, fija un marco para la clasificación de los comportamientos no verbales.

Desde la Antropología, se ha estudiado la Comunicación no verbal como una expresión cultural a partir de la cual se han establecido diferencias entre distintas etnias y nacionalidades; autores como Edwar Sapir (1949) y Margaret Mead en su libro "Male and Female" (1949) señalan el aprendizaje de los movimientos corporales, no como un fenómeno fortuito, sino como un conocimiento que se adquiere, se expresa, comprende, y al cual se responde con naturalidad desde la cultura.

En el año de 1942, Gregory Bateson asociado con Ray Birdwhistell, Edward T. Hall, Ervin Goffman y Paul Watzlawick entre otros, desde diferentes disciplinas como la psicología, antropología y sociología, conforman la Escuela de Palo Alto o el llamado "Colegio Invisible" como un espacio académico para estudiar la comunicación humana. Estos autores proponen como alternativa al modelo lineal de comunicación, trabajar desde el modelo circular retroactivo propuesto por Norbert Wiener (1948); donde el principal planteamiento para investigar la Comunicación no verbal es el enfoque sistémico, "en términos de niveles de complejidad, contextos múltiples y sistemas circulares" (Rizo, 2005), donde la comunicación humana se percibe como un todo y cada elemento constitutivo se relaciona entre sí y afecta a los demás.

Ray L. Birdwhistell, se concentró en el estudio de la cinésica, de hecho se considera el padre de esta disciplina; Edward T. Hall con su publicación "The silent language" (1959) realizó estudios alrededor de la proxémica. Paul Ekman y Albert Friesen propusieron un marco teórico para abordar los orígenes, uso y codificación de la conducta no verbal especializándose en las emociones expresadas a través del rostro con el postulado de la existencia de un vocabulario facial con algunas expresiones universales.

Por otro lado, Erving Goffman, abordó la Comunicación no verbal desde un marco más amplio, donde a partir de la propuesta del "Modelo dramatúrgico", permite hacer una liga con construcciones sociales agudas, tomando en cuenta las formas de socialización y normalización, es decir, la Comunicación no verbal como cultura interiorizada expresada a través de rituales, buenas maneras o máscaras sociales como actuaciones convincentes en la vida cotidiana. 
A través de las investigaciones en torno a este objeto de estudio, algunos de los autores ya mencionados como Birdwhistell o Ekman, en su búsqueda por unidades mínimas de significado, encontraron una analogía entre el movimiento del cuerpo y el lenguaje, principalmente en la dimensión de la función dentro de la comunicación humana. Por ejemplo, la similitud entre el uso de ciertos movimientos como signos de puntuación del lenguaje no verbal. En este sentido, la semiótica ha funcionado como herramienta para estructurar y analizar la información recabada. Formalmente, el semiólogo Thomas Sebeok en contraparte a lo que llamó la Antroposemiótica (estudio del comportamiento animal), fue el primero en realizar estudios en torno a la expresión humana no verbal. La revista especializada en semiótica "deSignis" en el año del 2002 dedicó un número a los gestos, sus sentidos y prácticas.

En la década de los sesenta y setenta se realizaron diversos estudios que contribuyeron a la comprensión de la Comunicación no verbal. Por ejemplo, en el año de 1956 el psiquiatra Jurgen Ruesch y el fotógrafo Weldon Kees realizaron un documento visual llamado "Nonverbal communication: notes on the visual perception of human relations"; y diversos autores como Argyle (1969), Dittmann (1972), Goldman-Eisler, Hess (1965, 1968), Kendon (1967,1970), Mehrabian (1976), Scheflen $(1963,1965)$ entre otros realizaron investigaciones en torno a este objeto de estudio.

Hasta que se creó "Fast, Body Language", un informe con alto impacto en el público y a partir del cual surgió una corriente de libros y artículos de revistas que simplificaban los estudios a resultados universales y comprensibles, para que el público en general los pudiera utilizar como medida infalible en relaciones interpersonales; incluso para conseguir con éxito pareja, trabajo o descubrir aspectos secretos y profundamente íntimos de las personas.

\section{LOS CAMINOS METODOLÓGICOS PARA EL ESTUDIO}

DE LA COMUNICACIÓN NO VERBAL

Como se mencionó anteriormente, la Comunicación no verbal se ha estudiado desde diferentes enfoques, metodologías y técnicas de acuerdo a la finalidad desde las distintas posturas epistemológicas. Sin embargo, antes de hacer una revisión en este sentido, resulta indispensable plantear el objeto de estudio con características propias y cierta complejidad para ser abordado, principalmente en tres sentidos: en relación a los sujetos de estudio, los sistemas de signos a trabajar y el rol del investigador en la experiencia empírica. 
En cuanto a los sujetos, cabe señalar el estado de descorporeización que se vive en la actualidad principalmente en la cultura occidental, donde aún persiste la concepción del ser en dos unidades diferentes e independientes entre sí: razón y cuerpo. La razón, asociada con el mundo objetivo, aceptado, a lo digno, lo valioso; el mundo de la inteligencia, del poder, que es motivo de respeto y que se concibe como la lógica que nos permite sobrevivir en la convivencia social. Por otro lado, el cuerpo vinculado con la sexualidad, lo mundano, con sensaciones y sentimientos, con la sensualidad y lo prohibido; como el espacio personal que guarda placeres, historias y vulnerabilidades. Por lo tanto, alrededor de esta dimensión corporal existen tabúes y prejuicios, incluso existe la tendencia por tratar de ocultar sus olores, sus secreciones, sus curvas, sus líneas, sus enfermedades, su expresión plena; como menciona Birdwhistell: "hasta los buenos olores, los gustos y contactos agradables suelen ser objeto de desconfianza" (citado en Davis, 1973). Otro aspecto importante para tomar en cuenta y que complejiza la investigación de este tema es la normalización, desde la visión de Erving Goffman y como descubrió Paul Ekman, las normas y reglas de etiqueta sociales pueden impedir la expresión plena de algunas expresiones corporales, donde la presión del orden social y la aceptación del otro pueden provocar cierto autocontrol en la expresión corporal y gestual de los sujetos para no romper con la dinámica de lo considerado "normal".

Planteados estos escenarios, podemos decir entonces que no somos totalmente conscientes de nuestra corporeidad y la expresión de la misma; la cantidad de formas, ritmos y posibilidades alrededor del movimiento corporal es inmensa, sutil y por esta razón la expresión corporal puede vivirse y actuarse de forma instintiva, inconsciente. Aquí se inserta otro aspecto importante en torno a la naturaleza de los signos, es decir su calidad de espontaneidad-individualidad así como su construcción desde la cultura, desde lo social.

Por otro lado, es importante considerar que un gesto o un movimiento puede tener diferentes significados dependiendo del lugar, la situación, el sujeto, la intención, relación con el discurso verbal, etcétera.

En línea con lo mencionado anteriormente y en relación al investigador, éste difícilmente puede comprender el sentido para los sujetos acerca de su cuerpo y su expresión desde una posición externa; sin embargo trabajar con el discurso a partir de técnicas tradicionales utilizadas por las ciencias sociales, implica que los sujetos construyan procesos complejos de autopercepción y autorreflexión de forma estructurada y lógica a través del lenguaje. 
Por otro lado, al momento de que el investigador intenta registrar lo observado, requiere una capacidad minuciosa de descripción y un alto nivel de sensibilidad, incluso entrenar y afinar la mirada para observar algunos movimientos mínimos; tomando en cuenta además, que no existen códigos que nos permitan realizar traducciones universales de movimiento para registrar lo que se observa.

Finalmente, existe un filtro de lenguaje tanto de los sujetos para hablar en torno a su cuerpo, como del investigador para registrarlo o interpretarlo.

Posiblemente como consecuencia de las consideraciones anteriores y por la naturaleza visual del objeto de estudio, la principal técnica de recolección de información se ha realizado a partir del levantamiento de imagen, es decir, con la filmación y análisis de películas de sujetos en interacción; por ejemplo, la investigación que realiza Kendon en "Studies in Human Greetings" (1972), donde se filmaron sesiones de saludos en una fiesta infantil.

Los psicólogos y psicoterapeutas, han analizado a los sujetos a través de filmaciones dentro de cámaras de Gessell o laboratorios principalmente en espacios académicos o durante terapias ya que el interés principal desde este enfoque es relacionar aspectos gestuales y corporales con la personalidad y/o la dimensión emotiva de los sujetos. Cabe mencionar que otras disciplinas como la Antropología o la Sociología a partir del enfoque sistémico han considerado que el desarrollo de la conducta humana en espacios recreados conlleva a situaciones forzadas donde los sujetos no se comportan ni expresan de forma plenamente honesta. Así, se ha optado por realizar las filmaciones en los contextos naturales de interacción de la vida cotidiana, ya que desde este abordaje epistemológico lo relevante es observar a los sujetos en interacción en un espacio y tiempo determinados con el fin de observar y registrar la relación entre la comunicación humana, el contexto y la cultura.

Sin embargo, a pesar de que la filmación ha sido la principal técnica de recolección de información para distintas disciplinas, la diferencia se encuentra en el tipo de análisis. Se ha realizado, principalmente desde la Psicología, el análisis a detalle, es decir cuadro por cuadro y en cámara lenta, con el fin de poder descubrir las reacciones específicas y sutiles de la infinidad de expresiones faciales y corporales de los sujetos en relación con su comportamiento, estados de ánimo o incluso psicopatías. A través de esta vía se lograron detectar tendencias o diferencias entre géneros y en algunos casos, se confrontó a pacientes con su imagen video grabada con el fin de realizar un proceso de autorreflexión acerca de su movimiento en relación al comportamiento dentro de un grupo. Otros 
han realizado el procesamiento de la información visual a nivel estadístico y analítico para descubrir las tendencias, un análisis de tipo cuantitativo.

El antropólogo Ray Birdwhistell, realizó el análisis de lo visto en los filmes a partir de una metodología llamada microanálisis, donde realizó una trascripción de la imagen a un sistema de códigos escrito. De esta forma destina una representación gráfica a cada micromovimiento tomando en cuenta su dirección y la parte del cuerpo en juego; así se registra todo lo que sucede en 24 cuadros por cada segundo de película, donde se verifican regularidades y repeticiones, no como unidades aisladas sino como un conjunto de signos que se construyen y toman sentido a partir de los diferentes contextos y culturas.

Birdwhistell, interesado en la comunicación humana desde la teoría sistémica, descubrió a partir de estas transcripciones gráficas, que existe una analogía a nivel estructural entre la kinesis y el lenguaje, realizó un análisis similar al que realizan los lingüistas estructurales con la lengua, distinguiendo planos semánticos, sintácticos y pragmáticos. Determinó entonces dos unidades fundamentales: kine como la unidad menor, un movimiento apenas perceptible, y kinemas entendidos como los movimientos mayores, portadores de sentido tomados en conjunto; lo equivalente al morfema lingüístico.

Por otro lado, Ekman en su preocupación por encontrar un método fiable para descifrar las expresiones, trabajó con Wallace Freisen y el psicólogo Silvan Tomkins para crear una especie de atlas del rostro llamado FAST (Facial Scoring Technique). El interés se centró en generar un sistema y un documento visual para categorizar las expresiones faciales y tener la capacidad de ligarlo con la dimensión emotiva sin el filtro del lenguaje del sujeto, evitando así las diferentes capacidades de verbalización de sentimientos o incluso la posibilidad de encontrarse con mentiras. Este documento cataloga las expresiones faciales mediante fotografías dividiendo al rostro en tres zonas: la zona de cejas y frente; la zona de ojos, párpados y nariz; y mejilla, boca, mentón y mandíbula. De esta forma abarca un mínimo de seis efectos faciales primarios y treinta y tres mezclas ligadas con sentimientos, se consideró entonces al rostro como una de las zonas más expresivas del ser humano. Por otro lado, estos autores también realizaron un sistema de clasificación de los comportamientos no verbales a partir de su función, similar a lo realizado en estudios de lenguaje. ${ }^{1}$

Otra técnica utilizada ha sido la pupilometría, también desde la psicología

${ }^{1}$ Freisen y Ekman (1976) proponen una clasificación de los movimientos corporales dividiéndolos en: emblemáticos, ilustradores, muestras de afecto, reguladores y adaptadores. 
y aplicada por Eckard Hess. En este caso se pedía a los sujetos que miraran a través de un lente, donde una cámara cinematográfica filmaba los ojos mientras se mostraban diapositivas con imágenes con diferentes connotaciones. Así, la pupila humana reaccionaba a las imágenes, dilatándose cuando las imágenes eran placenteras o contrayéndose cuando eran desagradables. Con el estudio de las respuestas pupilares se pudieron determinar reacciones a nivel anatómico que reflejan la dimensión emotiva ante estímulos externos.

Para realizar investigación en torno a la hanóptica o estudios en relación a la interacción táctil, el psicólogo Sidney Jourard trabajó con mapas, pidiendo a sus alumnos que mostraran las zonas del cuerpo tocadas con mayor frecuencia por su madre u otras personas, encontrando así diferencias relevantes en la interacción con diferentes vínculos y géneros.

Casi de forma aislada, en México, Jesús Becerra realizó un estudio alrededor de la proxémica a través del registro poligráfico, "el estudio pretendió establecer si había correspondencia entre las distancias en que son recibidos los estímulos de presencia corporal y los derivables de una conversación, respecto a los ritmos de trabajo cerebral, muscular facial y cardiaco." ${ }^{2}$

A manera de síntesis, por un lado se puede afirmar que la psicología ha sido la disciplina que ha mostrado mayor interés y quien ha aportado más herramientas técnicas al estudio de la Comunicación no verbal, con la finalidad de conectar la expresión corporal con comportamientos, emociones o patologías humanas. En general, los estudios en torno a la Comunicación no verbal se han desarrollado principalmente para atender los siguientes objetivos:

1) Conocer, descifrar y ordenar el lenguaje corporal en sus diferentes dimensiones $^{3}$ y funciones principalmente en relación con el discurso.

2) Relacionar la expresión corporal con la cultura y el contexto.

3) Relacionar la Comunicación no verbal con la dimensión psíquica y emotiva de los sujetos.

Sin embargo, debido a la relativa novedad de la investigación en torno a la Comunicación no Verbal se puede decir que hasta el momento y de forma

\footnotetext{
${ }^{2}$ La hipótesis central del trabajo propone que el polígrafo efectivamente registraría respuestas cognoscitivas y emocionales en los sujetos. Catálogo de Documentación en Ciencias de la Comunicación (CC-DOC). Recuperado e1 5 de febrero del 2006, de http://148.201.94.6/ biblioteca/bibdigital/desc/ccdoc/

${ }^{3}$ Según Knapp (1982): kinésica, proxémica, conducta táctil, características físicas, paralenguaje, relación objetos y con el entorno.
} 
mayoritaria, los estudios se han centrado en sujetos principalmente de dos esferas: estudiantes de universidad y personas en terapia, en ambos casos con énfasis en la cultura norteamericana. Lo anterior, tiene que ver con el origen de los autores interesados en el tema y el espacio profesional con el cual tienen contacto cercano. Cabe señalar el reducido número de investigaciones en Latinoamérica y en México en torno a este objeto de estudio ${ }^{4}$, antes de iniciar una discusión en torno a la metodología ideónea para realizar proyectos de investigación al respecto, cabría reflexionar la falta de planteamientos en este tema, motivo de otro posible proyecto.

Si bien esta disciplina incipiente ha arrojado información sumamente valiosa y así como ha abierto un mundo poco conocido y grandes posibilidades de estudio, aún quedan algunos cabos sueltos en torno a las metodologías utilizadas, independientemente del enfoque desde donde se han diseñado. Por ejemplo, en el caso de las filmaciones, la recreación de las situaciones en espacios artificiales puede afectar la expresión natural de las conductas y expresiones de los sujetos; en algunas ocasiones el análisis se ha concentrado sólo en algunas unidades de movimiento, aislando otras que conforman la totalidad de la conducta. En general los resultados se ubican en un plano descriptivo, donde la calidad de la descripción se centra únicamente en la capacidad del investigador para distinguir movimientos sumamente agudos y muchas veces con interpretaciones supuestas sin incluir la perspectiva de los sujetos en cuestión. En relación al microanálisis, la traducción gráfica de cada mínimo movimiento requiere un arduo esfuerzo, Ray Birdwhistell mencionó haber invertido una hora de análisis por un segundo de película; más allá de la inversión en tiempo y trabajo, cabe cuestionar si la naturaleza de estos sistemas simbólicos es la misma al lenguaje y así, su análisis. Como menciona Lucrecia Escudero (2002, p. 9):

"Los gestos, prisioneros durante décadas en la ortopedia de la analogía con el lenguaje y su búsqueda metodológica de unidades mínimas de significación, leídos sólo a partir de de un sistema de unidades discretas y combinables, son el caso testigo de cómo la primera semiología de corte estructuralista puede constituirse como tal

${ }^{4}$ En el Catálogo de Documentación en Ciencias de la Comunicación se encontraron 8 textos en torno a la Comunicación no verbal: 1 mencionado anteriormente con una propuesta metodológica; 4 que realizan revisiones teóricas, 2 revisiones de casos donde tocan esta dimensión: cultura organizacional y experiencia amorosa; y 1 como reflexión de esta vía de comunicación ante el desarrollo de nuevas tecnologías. (idem Recuperado e1 5 de febrero del 2006) 
expulsando precisamente a aquellos objetos difícilmente modelizables y presentando como posición epistemológica la analogía y metáfora, del cuerpo como lenguaje para concluir que los gestos son sistemas simbólicos no lingüísticos.”

En el caso del catálogo de expresiones del rostro de Ekman y Friesen, FAST, existen algunos riesgos al no distinguir, por ejemplo una expresión sincera de una falsa a pesar del entrenamiento de la mirada, o confundir emociones mezcladas como una situación de ira ligada con temor, o ignorar la significación individual de diferentes expresiones. Para evitar este tipo de riesgos y en línea con la mirada sistémica, se tendrían entonces que desarrollar diferentes catálogos de acuerdo a distintas situaciones, espacios, culturas y según diferentes los sujetos, tomando en cuenta toda la expresión corporal e incluso con la retroalimentación de las personas. Por otro lado, la pupilometría resulta un sistema poco práctico en situaciones cotidianas.

Finalmente, cabe cuestionar si los sujetos en la vida cotidiana somos capaces de registrar, comprender y reaccionar con tal precisión a la minuciosidad de información que han arrojado estas investigaciones acerca de la Comunicación no verbal de los sujetos y por otro lado, si los investigadores tienen la suficiente sensibilidad de registrar y determinar significado a las diferentes expresiones localizadas.

Propuesta de metodología para estudiar la Comunicación no verbal

Después de haber planteado el estado del arte en torno a las diferentes posturas metodológicas, finalmente la intención es plantear una propuesta en este sentido para investigar el objeto de estudio en cuestión desde la disciplina que debiera ser la especialista -la Comunicación-, y que paradójicamente aún se encuentra en un estado incipiente en comparación con otras.

A partir de la reflexión anterior, así como de la observación de Lucrecia Escudero (quien metafóricamente plantea el estudio de los gestos como una prisión de la analogía con el lenguaje) y Patricia Magli (2002, p. 38): "el gesto no sólo informa como la palabra, también actúa, análogamente la comunicación a través del cuerpo también tiene funciones preformativas en las relaciones interpersonales"; cabe replantear la forma de abordar la Comunicación no verbal y entenderla con una naturaleza en sí para después plantear propiamente la metodología. De acuerdo con la definición propuesta en un inicio, concebirla entonces como un fenómeno complejo, un proceso humano de comunicación 
es decir, social, dinámico, en constante significación y adaptación con formas de producción de sentido propias. En este sentido, a continuación se proponen algunos ejes básicos en relación a este objeto de estudio desde la mirada de la Comunicación:

1) Considerar la Comunicación no verbal intrínseca de la comunicación humana.

2) Conceptualizar a la Comunicación no verbal como un sistema, es decir, entender que todos los elementos que la constituyen se encuentran relacionados construyendo una dinámica y lógica propias, y que la actuación o modificación de alguno de ellos va a afectar a los demás. Es decir, comprender la Comunicación no verbal como un todo (no unidades mínimas independientes), donde se debe registrar, analizar y comprender ese todo en conjunto.

3) Tomar en cuenta la naturaleza comunicativa de la comunicación no verbal, no obviar que la expresión corporal comunica de forma constante, incluso sin movimiento.

4) Considerar la naturaleza metacomunicacional, es decir, el uso del lenguaje para hablar acerca del lenguaje mismo.

5) Conceptualizar la Comunicación no verbal como habitus, lo que Bourdieu denomina hexis corporal 5 , "donde el cuerpo funciona como estructura mnemotécnica que retiene y reproduce las estructuras objetivas socializadas y aprehendidas" (Bordieu, 19876). En este mismo sentido, considerar los capitales de los sujetos, entendidos como recursos potenciales, posesiones definidas que determinan el desarrollo en el espacio social, Pierre Bordieu (1987, p. 106) plantea cuatro capitales presentes en el individuo: capital cultural, capital económico, capital social y capital simbólico. (Ver nota 1)

${ }^{5}$ Según Bourdieu, "el porte, los gestos, la manera de presentar, trabajar el cuerpo, definen una fisonomía social en los que están depositadas las disposiciones más básicas y vitales del habitus, donde las personas expresan el aprendizaje de su lugar en el mundo y su manera objetiva de ser en el mundo. En la cara, los brazos y las piernas están depositados imperativos y valores sociales aprendidos en el pasado y ahora hechos cuerpo." (Citado en La identidad que otorga la posición. El caso de los bolivianos en Jujuy. Un análisis desde la perspectiva de Pierre Bourdieu”, texto de Juan Armando Guzmán. Recuperado de: http://www.elcolegiodesantiago.com.ar/ponencia_2004-25.htm)

${ }^{6}$ Bourdieu (citado en García, A.). (1987). Fragmentos seleccionados y escanadeos del Habitus según Bourdieu. 
6) Ubicar y conceptualizar la Comunicación no verbal como parte y producto de la cultura, donde para comprender la construcción de sentido de los sujetos de estudio, se deben ubicar y contextualizar en un tiempo, espacio y lógica determinada a partir de donde se construye el significado, que además puede variar de acuerdo a la situación y al sujeto.

7) Tomar en cuenta la dimensión histórica, donde los rasgos y la expresión corporal se van construyendo y transformando a lo largo del tiempo, así mismo la significación, apropiación y construcción de sentido. El habitus reintroduce la dimensión histórica como estructura generativa que asegura la actuación del pasado en el presente. ${ }^{7}$

8) Trabajar con procesos de comunicación en lugares y situaciones cotidianas que se acerquen a conductas naturales, no forzadas; y así considerar también las expresiones espontáneas e individuales.

9) Tomar en cuenta las reglas de etiqueta construidas a partir del orden social como parte de las normas sociales adaptadas e incorporadas en la vida cotidiana, desde el enfoque dramatúrgico propuesto por Goffman.

\section{Propuesta tÉcnica}

A partir de los planteamientos mencionados anteriormente para abordar el objeto de estudio, a continuación y desde una perspectiva hermeneútica se propone lo relacionado con la técnica propiamente. Esta metodología se está poniendo en práctica actualmente en un proyecto de investigación ${ }^{8}$ que a grandes rasgos pretende conocer los factores involucrados en la construcción del lenguaje corporal en alumnos universitarios, así como la forma en que se desarrolla y expresa en diferentes espacios y situaciones de interacción. Cabe

\footnotetext{
${ }^{7}$ Bourdieu afirma que el habitus produce prácticas conformes a los esquemas engendrados por la historia y asegura la presencia activa de las experiencias pasadas que, depositadas en cada organismo bajo la forma de esquemas de percepción, de pensamiento y de acción, tienden -de forma más segura que todas las reglas formales y todas las normas explícitas- a garantizar la conformidad de las prácticas y su constancia en el tiempo. (idem)

${ }^{8}$ Este proyecto se desarrolla en el marco de la Universidad Intercontinental con el nombre de "La comunicación silenciosa: el cuerpo hablando". Por otro lado, se está haciendo una labor conjunta para realizar adaptaciones con el fin de aplicar la metodología en un proyecto conjunto con la Universidad Autónoma de la Ciudad de México: "Mediaciones lingüísticas de autopercepción y estrategias de autorrepresentación sobre el cuerpo femenino" con Vivian Romeu y Cynthia Pech como responsables.
} 
señalar la conveniencia de aplicación dentro del contexto mexicano al entender la expresión corporal como un constructo desde lo social así como de la historia individual de los sujetos, por esta razón se plantea el uso de técnicas que combinan las dos dimensiones: la observación participante y biografías corporales, así como talleres de movimiento como área de intervención desde la Comunicación. Finalmente al ser el lenguaje el medio para que los sujetos reflexionen en torno al uso y significaciones del cuerpo, así como para que el investigador describa cómo éste se expresa, resulta pertinente aplicar una metodología que contemple el discurso del contexto donde se desarrolla la interacción.

\section{OBSERVACIÓN PARTICIPANTE}

En un primer nivel, esta técnica funciona para tener una mirada general, un primer acercamiento para distinguir características de la expresión corporal así como las diferencias generadas a partir de distintas lógicas de acuerdo a los sistemas considerados. Se plantea como participante en el sentido de que el investigador "participa", más que con entrevistas insitu, al compartir simbolismos o significaciones de expresiones de una misma cultura para describir situaciones de interacción. A continuación se plantean algunos aspectos para tomar en cuenta al investigar la Comunicación no verbal de los sujetos dentro de un entorno social, así como una vía para estructurar los datos arrojados por la investigación de campo. Estos aspectos están divididos en primarios, secundarios y terciarios:

\section{a) Aspectos primarios}

Se refieren a las características que permiten plantear las condiciones contextuales de los actores sociales y que permiten comprender la relación con el entorno. Entre ellos destacan la nacionalidad u origen, el género, la edad y estilo de vida.

- Nacionalidad/origen: diferentes investigaciones han arrojado información acerca de los diferentes códigos de Comunicación no verbal a partir del origen de los sujetos, desde la antropología por ejemplo. De igual forma en que se hablan diferentes idiomas, también existen diferentes lenguajes corporales íntimamente relacionados, por ejemplo la gesticulación exagerada de los italianos con las formas elegantes y sencillas de los ingleses. 
- Género: se ha comprobado por investigaciones previas ${ }^{9}$ que culturalmente los sujetos apropian desde la cultura elementos de lenguaje corporal. Desde el entorno familiar o religioso por ejemplo, se enseñan y apropian formas de comportarse a partir del género; se fortalecen o inhiben códigos femeninos o masculinos culturalmente aceptados.

- Edad: la fisonomía se va transformando conforme el desarrollo de vida de los sujetos y estos cambios a nivel anatómico implican formas de apropiación, adaptación o rechazo de acuerdo a criterios (conscientes o no) de la persona. Por lo tanto, estos cambios a nivel anatómico también tienen que ver con la significación e imaginario de diferentes etapas de vida de los sujetos.

- Estilo de vida: entendido como "producto sistemático de los habitus que, percibidos en sus mutuas relaciones según los esquemas del habitus, devienen sistemas de signos socialmente calificados". (Bourdieu, 1979, p. 171). Es decir, identificar elementos visibles, signos que nos hablen del habitus de grupo, así como acerca los capitales de los sujetos (hasta donde puedan ser observables). Pensemos, por ejemplo, las posibles diferencias de códigos entre tribus juveniles o grupos religiosos, deportistas, artistas, etcétera. Incluso una misma persona puede desarrollar lenguajes diferentes en grupos distintos.

b) Aspectos secundarios

Denominados así no por tener menor relevancia, sino por implicar un segundo nivel de especificidad. Los aspectos secundarios son:

- Marco: a partir de lo que Goffman entiende como dispositivo cognitivo y práctica de organización de la experiencia social, es decir no sólo se debe tomar en cuenta como referencia espacial, sino el marco sistémico a partir de donde se genera una lógica específica de normas y conductas sociales con implicaciones simbólicas en procesos comunicativos.

- Ambiente: del lugar, es decir, tener presente cómo el tipo de iluminación, materialidades, personas presentes, olor, ruido y otras condiciones del espacio influyen en los momentos de interacción y construye una atmósfera determinada.

- Situación: es la razón de ser de la interacción, el motivo por el cual los sujetos se reúnen y establecen procesos comunicativos, se da en un momento

${ }^{9}$ El estudio del cuerpo con la perspectiva de género ha sido abordado en México por autores como: Lorena Zamora (2000), Margarita Baz (1994), Marta Lamas (1994) y Katya Mandoki (2003). 
determinado y al momento en que se desarrolla también se puede ir transformando.

- Interacción con otras personas: específicamente los sujetos involucrados en los procesos de comunicación, con los cuales se establece relación.

\section{c) Aspectos terciarios}

Se refiere al nivel más fino de la observación, la descripción detallada de la Comunicación no verbal en momentos de interacción. A continuación se propone una guía de elementos a observar que también funciona como una forma para estructurar la información en cuanto a la expresión corporal.

- Características físicas: se refiere a los rasgos de expresión de los sujetos. Es relevante ya que pueden ser elementos que determinen la interacción con los otros. Se pueden ubicar dos tipos: estáticos y evolutivos. Dentro de los estáticos, como características que permanecen constantes, se pueden ubicar el color de ojos, la forma de cara, tono de piel o el tipo de manos. Dentro de los evolutivos como el cambio o adaptación de las características físicas se pueden ubicar la coloración de los dientes, la constitución, altura, disposición del cuero cabelludo, acne, cicatrices, arrugas, etcétera.

- Posturas: colocación y disposición general del cuerpo, por ejemplo de los hombros, el portar la espalda recta o encorvada.

- Gestos: movimientos y expresiones faciales alrededor de tres secciones del rostro: el área de los ojos, la nariz y la boca.

- Mirada: como un elemento específico por la relevancia durante la interacción, por ejemplo el lugar hacia donde se mira, la intención o tipo de mirada en los procesos comunicativos.

- Kinésica: entendida como el estudio de los movimientos del cuerpo donde cabe resaltar el ritmo, la calidad de movimiento, la intención o uso de la expresión corporal. (ver nota al pie 1)

- Paralenguaje: se refiere a la forma de decir. Según Trager (1958) se ubican diferentes componentes:

o Cualidades de la voz: como el control del ritmo, tiempo, articulación, resonancia, control de la glotis y control labial de la voz.

o Vocalizaciones: donde se encuentran los caracterizadores vocales como risa, llanto, suspiros; los cualificadores vocales donde entra la intensidad de la voz, la inflexión del tono y la extensión al articular las palabras; y finalmente las segregaciones vocales como las muletillas, "mmm", "aha", los silencios, errores al hablar, etcétera. 
- Interacción con objetos: es decir la manipulación de artefactos como estímulos en procesos comunicativos, como arreglarse los lentes al hablar u observar constantemente el reloj.

- Conducta táctil: como el contacto físico con los otros en diversas situaciones, por ejemplo el abrazo o la ausencia de contacto en la situación de saludo.

- Vestimenta: la ropa habla muchas veces del estilo de vida del sujeto, la forma como se percibe y la relación con su contexto. Por ejemplo una persona que hace ejercicio y se siente cómoda con su cuerpo puede utilizar ropa deportiva ajustada a su figura en la vida cotidiana. Es conveniente considerar entonces el tipo de ropa utilizada, si está a la moda o no, la gama de colores, tipo de telas; preguntarse qué dice la vestimenta de la persona. $\mathrm{Y}$ aunque no estrictamente sea vestimenta, las formas para "vestir" o arreglar el cabello.

- Proxémica: entendida como el estudio del uso y percepción del espacio social y personal (Knapp, 1982, p. 25), es decir cómo la gente dispone del espacio personal, cómo usa el espacio en la interacción con otros y cómo responde a la disposición espacial de los sujetos y objetos en el espacio.

\section{Biografías CORPORALES}

Esta técnica propone retomar al lenguaje como uno de los actores principales a partir de lo que tradicionalmente se conoce como historia de vida e historia oral temática, realizando los ajustes necesarios para el objeto de estudio en cuestión, razón por la cual se denomina "Biografía Corporal", entendida como una mezcla entre las técnicas anteriormente mencionadas donde se trabaja en diferentes niveles:

a) Principalmente a través de la subjetividad de los sujetos podemos conocer la construcción de sentido y significación en torno a la expresión corporal para complementar la observación realizada por el investigador

b) El cuerpo como eje temático en cuestión de la relación con él mismo y sus significaciones, así como el uso y prácticas que se le da en la vida cotidiana y en momentos de interacción. Es decir, los valores y las convenciones sociales aprendidas hechas cuerpo, lo referente a la construcción del hexis corporal.

c) La reconstrucción histórica de la vida del sujeto, es decir el trabajo con el recuerdo, principalmente enfocado a su relación con el cuerpo, que permite contextualizar y ubicar transformaciones en la construcción de sentido para los sujetos. 
d) Influencias del entorno relacionado para construir o aprehender la comunicación no verbal a través del tiempo. Es decir a partir de características físicas propias, conocer cómo ha sido el proceso de adaptación y uso del cuerpo, como señales de cortesía aprendidas desde la educación en casa, las normas de género u otros aspectos desde la cultura, que permiten saber cómo reaccionar y comportarse en diferentes situaciones. Como, por ejemplo, el tener senos grandes y en la vida cotidiana esconderlos o mostrarlos.

Si bien anteriormente se planteó la dificultad de trabajar con el discurso de los sujetos por las implicaciones de orden y estructuración de una dimensión de la persona a veces olvidada, considero que utilizar el lenguaje como un mediador entre el mundo interno y el externo, tiene varias implicaciones positivas. Por un lado, involucrar al otro para comprender un fenómeno que tiene que ver con su intimidad, autopercepción y subjetividad, como es la relación con su cuerpo; por otro lado el nivel de reflexividad requerido de parte de los sujetos de estudio al recordar, reconstruir y verbalizar en torno a una dimensión de ellos mismos, comprendido además desde la lógica natural del lenguaje, "centrada en los procesos de argumentación en el que la sujeto argumenta a favor o en contra sus creencias y experiencias" (Pech, C. \& Romeu V., 2006), en este caso en torno su propio cuerpo.

Así, se apuesta a estudiar la Comunicación verbal desde una perspectiva diferente con posibilidad de construir conocimiento con mayor complejidad y profundidad al integrar al sujeto en un proceso activo de configuración de sí mismo y en torno al objeto de estudio.

\section{TALLERES DE MOVIMIENTO}

Finalmente los talleres de movimiento más que una técnica de investigación en sentido estricto, se proponen como un espacio para verter los conocimientos ya sistematizados de las técnicas ya mencionadas anteriormente. Es decir, como parte de la metodología en la dimensión de la investigación-acción, donde se plantea un escenario para explorar, intervenir y probar nuevas posibilidades con los datos obtenidos, incluso para complementar o verificar la información ya sistematizada con una gama de posibilidades como por ejemplo:

a) Intensificar la conciencia en torno a la expresión corporal en procesos de comunicación. 
b) Identificar códigos que puedan mejorar procesos comunicativos en situaciones específicas.

c) Generar mensajes construidos sólidamente tomando en cuenta la dimensión verbal y no verbal.

d) O conocer con mayor profundidad los sujetos de estudio o que éstos obtengan mayor conocimiento entre sí como vía para mejorar procesos comunicativos.

Finalmente, con esta propuesta metodológica cabe la posibilidad de cruzar tres diferentes momentos y puntos de vista, la del pasado con la construcción y significación histórica desde el sujeto de estudio a partir de su discurso, la del presente desde el punto de vista del investigador a través de la observación en torno a la Comunicación no verbal en uno o varios escenarios y desde el punto de vista del sujeto a través del discurso actual; y la apuesta hacia el futuro a través de la intervención en procesos de comunicación, mediante los talleres de movimiento.

La intención es traspasar el plano descriptivo, ya que si bien este nivel de información ha sido enriquecedor como vía para tener un primer acercamiento y dimensionar todas las posibilidades de este objeto de estudio; también es importante que las investigaciones a futuro en torno a la Comunicación no verbal trasciendan los catálogos formales, estáticos, muchas veces arbitrarios. De esta forma se pretende dar un papel más activo a los sujetos para comprender el fenómeno también desde su punto de vista con la posibilidad de cruzar subjetividades.

Aún queda una gama de áreas por optimizar, por ejemplo lograr el nivel de confianza y reflexividad necesarias para que los individuos generen discurso en torno a su mundo íntimo, encontrar más fácilmente sujetos dispuestos a compartirlo; la capacidad como investigador de registrar a detalle o la posibilidad de construir un lenguaje de traducción corpórea universal. Estas son sólo unas ideas.

Tal vez alguno de los aspectos mencionados, posiblemente sin respuestas plenas y concisas tiene que ver con el reducido número de estudios en torno al tema en América Latina, a lo mejor se considera un tema "poco académico" o se vislumbran aplicaciones poco éticas o simplemente genera poco interés. Sea cual fuere la respuesta, me parece que como comunicólogos e interventores de la realidad es clave mirar a los sujetos y comprenderlos con toda su complejidad, sin mermar la corporeidad y sus implicaciones en la construcción de identidad, 
subjetividad y al momento de interactuar, de comunicarse con los otros. De esta forma se abre una posibilidad para seguir construyendo conocimiento en torno al objeto de estudio y a la forma de abordarlo. Desde la perspectiva latinoamericana y mexicana aún falta un largo camino por recorrer, éste es sólo el inicio de uno posible que se empieza a construir.

\section{Notas}

1. El capital cultural, es el conjunto de "factores eficientes", bienes, propiedades que permiten a sus poseedores ejercer un poder en alguna área de las prácticas culturales. Pueden ser en el conocimiento, la educación, el lenguaje, la ciencia, la estética, los gustos, en general, en los modos de apropiación de los objetos culturales legítimos con que cuenta una sociedad. El capital social es la suma de recursos, actuales o potenciales, correspondientes a un individuo o grupo en virtud de que estos poseen una red duradera de relaciones, de conocimientos y reconocimientos mutuos, más o menos institucionalizados. Se trata del reconocimiento que permite a las personas movilizar en su favor y en momentos apoyos, garantías, influencias, que le proporcionan algún bien material o simbólico. El capital simbólico es una propiedad cualquiera, fuerza física, belleza, riqueza, valor, que percibido por los agentes sociales dotados de las categorías de percepción que les permite percibir y reconocerla deviene simbólicamente. El capital económico en relación con el concepto de clase. Posesión de capacidades, poderes y propiedades sociales similares en torno a las cuales es posible teóricamente reunir a grupos, creando un efecto de condensación y diferenciación respecto a otras condiciones de existencia (clases). (citado en Mazzoni, M. El habitus y el espacio de los estilos de vida. Culto al Cuerpo.)

\section{BiBLIOGRAFÍA}

Baz, M. (1994). Enigmas de la subjetividad: Un análisis del discurso, Versión Estudios de Comunicación y Política, No. 4. México: UAM Xochimilco.

Birdwhistell. (1970). Kinesic and Context: Essays on Body Motion Communication. Philadelphia: University of Pennsylvania Press.

Bourdieu, P. (1979). La distinción. Criterios y bases sociales del gusto. México: Ed. Taurus.

Bourdieu, P. (1987). Cosas Dichas. España: Ed. Gedisa.

Davis, F. (1973). La comunicación no verbal. México: Alianza Editorial.

DeVito, A. Humman Communication. The basic course. Estados Unidos: Ed. Harper and Row. 
Galindo, J. (coord.). (1998). Técnicas de investigación en sociedad, cultura y comnicación. México: Addison Wesley-Longman.

García, A. Fragmentos seleccionados y escaneados. El habitus según Pierre Bordieu. Recuperado el 5 de mayo del 2006, de http://www.univalle.edu.co/ -cognitiv/ archivos/grupo\%20cultura/Otros\%20dopcumentos/BOURDIEUHABITUS. pdf

Guzmán, J.A. La identidad que otorga la posición: El caso de los bolivianos en Jujuy. Un análisis desde la perspectiva de Pierre Bourdieu. Recuperado el 5 de mayo del 2006, de http://www.elcolegiodesantiago.com.ar/ponencia_2004-25.htm

Joseph, I. (1999). Erving Goffman y la microsociología. España: Ed. Gedisa

Karam, T. Introducción a la Semiótica. Portal de Comunicología (GUCOM). Recuperado el 20 de abril del 2006 de http://www.geocities.com/comunicologiaposible

Knapp, M. (1982). La Comunicación no verbal. El cuerpo y el entorno. México: Ed. Paidós.

Macías, N. (2005). Teoría de sistemas. México: En prensa.

Mandoki, K. (2003). Cuerpo, lugary discurso. Reflexiones en torno a la producción del poder, Versión Estudios de Comunicación y Política, No. 13. México: UAM Xochimilco.

Marc y Picard (1988). La interacción social: Cultura, instituciones y comunicación. Barcelona: Ed. Paidos.

Mazzoni, M. El habitus y el espacio de los estilos de vida: Culto al Cuerpo. Recuperado el 14 de mayo del 2006, de http://www.alipso.com/monografias/bourdieu_practicas_sociales/

Rector, M., Magli, P. \& Sebeok. (2002, octubre). Los gestos/sentidos y prácticas (vol. 3). Designis, España: Ed. Gedisa.

Pech, C. \& Romeu, V. (2006). Mediaciones lingüistias de autopercepción y estrategias de autorrepresentación sobre el cuerpo femenino, Memorias del Encuentro Nacional AMIC 2006, Mesa Comunicación y Género.

Rizo, M. (2004). El camino hacia la "Nueva Comunicación". Breve apunte sobre las aportaciones de la Escuela de Palo Alto", Razón y Palabra.

Varios. (2005). La comunicación interpersonal. Introducción a sus aspectos teóricos, metodológicos y empiricos. México: Universidad Autónoma de la Ciudad de México.

Catálogo de Documentación en Ciencias de la Comunicación (CC-DOC). Recuperado el 5 de febrero del 2006 de http://148.201.94.6/biblioteca/bibdigital/desc/ ccdocl 